\title{
Dielectric Breakdown Patterns in Thin Layers of Oils
}

\author{
RU-PIN PAN, ${ }^{+}$CHIA-RONG SHEU ${ }^{+}$and L. LAM ${ }^{*}$ \\ + Department of Electrophysics, National Chiao Tung University, \\ Hsinchu, Taiwan 30050, R.O.C. \\ * Nonlinear Physics Group, Department of Physics, \\ San Jose State University, San Jose, CA 95192-0106, U.S.A.
}

\begin{abstract}
Experimental dielectric breakdown patterns are generated in thin cells of oils. Both mineral oil and olive oil are used. The cell is $6 \mu \mathrm{m}$ thick and consists of two glass plates with conductive coating on the inner surfaces. A dc voltage across the cell is either increased gradually to above the threshold or applied with a fixed value above the threshold. Different types of patterns due to chemical reactions occurring at the inner surfaces are left behind after the dielectric breakdown process takes place. These patterns consist of filaments with wiggling or winding, depending on the experimental conditions. Time variations of the voltage and current are recorded during and after the discharge process. Some of these patterns are compared with those generated by the active walker model. The physical processes behind these patterns are discussed.
\end{abstract}

\section{INTRODUCTION}

Pattern formation [1-3] is an important and integral part of nonlinear physics [4]. While universal principles underlying pattern formation, if any, remain to be discovered, many particular pattern-forming systems have been studied in detail. In this regard, the case of dielectric breakdown (DB) is somewhat peculiar. While there exist an abundant amount of experimental data on DB patterns in solids [5], they have never become the focus of the pattern formation community. On the other hand, DB in compressed $\mathrm{SF}_{6}$ gas did play a crucial role in motivating the now famous dielectric breakdown model [6]; but DB patterns in 
gases never caught on after that.

Dielectric breakdown in liquids $[7,8]$ did not do any better. However some interesting developments have happened recently in this case. In the past, $\mathrm{DB}$ in liquids were studied experimentally by using two electrodes immersed in the liquid, and the DB track patterns were photographed. The electrodes may be spherical or in other shapes, or, e.g., of the point-plane configuration [9]. In all these cases, DB occurs in three dimensions and the tracks cannot be preserved permanently for further study after the experiment. Three years ago, a new type of DB experiment was initiated by Lam et al [10], in which a thin layer of liquid (or liquid crystal) was placed between two parallel semi-transparent glass plates with conductive coating on the inner surfaces. After the DB process is completed, different types of filamentary patterns [10-12] due to chemical reaction occurring at the inner surfaces of the cell are left behind. The system is quasi-twodimensional. To simulate these DB patterns an active walker model (AWM) was proposed [13], which turns out to be applicable to many other complex systems $[14,15]$.

In this paper, experiments on the DB of oils with a setup similar to that in Ref. [10] are reported. Conditions under which each type of DB patterns occurs are carefully recorded. Furthermore, the time dependences of the voltage and current during and after the DB process are measured. The physical processes are discussed. Some of these DB patterns are compared with those generated by the AWM. Discussions on a related experiment by Atten and Saker [16] are included.

\section{EXPERIMENTAL METHODS}

A glass plate with conductive indium tin oxide coating is cut to form $3.5 \mathrm{~cm} \times 4.00 \mathrm{~cm}$ slides, which are then cleaned. Either olive oil or mineral oil is put between two glass slides with the coating on the two inner surfaces. To control the cell thickness, two pieces of $6 \mu \mathrm{m}$ thick mylar with shapes "[" and "]" are put between the glasses along two opposite edges, forming a rectangular enclosure with two openings at opposite ends. The openings allow the oil and/or vapor to escape during the DB process, preventing any possible explosion of cell. Clamps are used to hold the cell together. When a cell is made, it is checked to make sure that there is no electrical short before the sample is used for experiments.

The experimental setup is shown in Fig.1. Voltage is applied across the cell using conductive thin wires attached to the coated inner surfaces. Two types of experiments, classified according to the method of applying voltage across the cell, are performed: (i) in the increasing-voltage method (IVM), the switch is closed first; a dc voltage is increased gradually from zero to the threshold at which DB occurs and then is maintained at constant; and (ii) in the fixed-voltage method (FVM), a dc voltage above the breakdown threshold is turned on before the switch is closed. The voltage is measured by a digital multimeter. In both methods, a Hewlett-Packard 6448B power supply is used. A digital storage oscilloscope is used to record the 


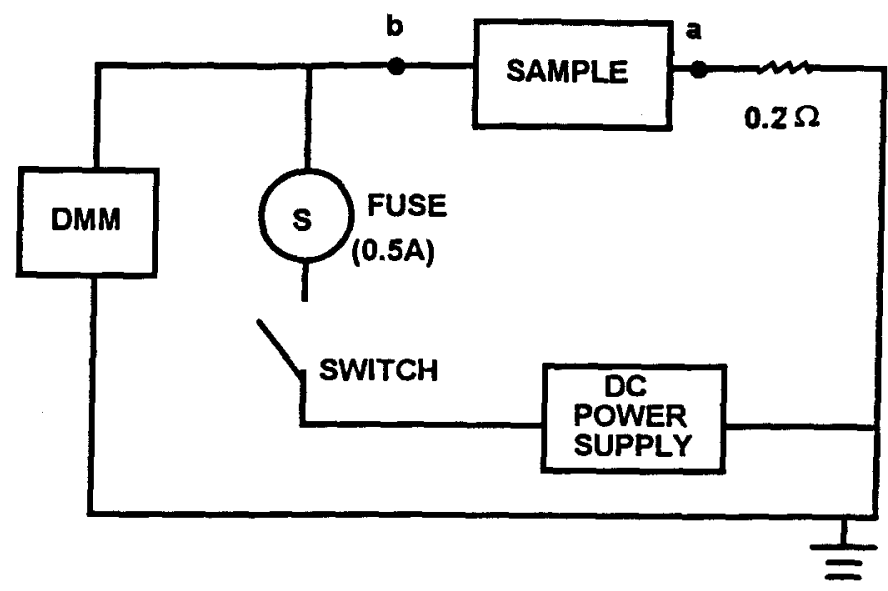

Fig. 1. The experimental setup. DMM: digital multimeter. A digital storage oscilloscope is used to measure, respectively, the voltages at point $a$ and point $b$, both relative to the ground. The voltage at point $b$ can give us the current through the cell, and the voltage difference between point $a$ and point $b$ is the voltage across the cell.

time variations of voltage and current across the cell.

During the breakdown discharge process a chemical reaction takes place. This is evidenced by the black ashes on the filaments--the "burnt" tracks--forming the discharge pattern left permanently on the inner glass surfaces, and the air bubbles created in the cell, which seems to occur along the filaments when they are first created.

The fuse is used to protect the equipment. Either a $1 \mathrm{~A}$ or $0.5 \mathrm{~A}$ fuse is used. Discharge stops under one of three conditions: when the voltage is off, in some cases when the discharge reaches the cell boundary, or when the fuse is melted.

\section{DIELECTRIC BREAKDOWN PATTERNS}

In the IVM, the breakdown voltage $\mathrm{V}_{\mathrm{c}}$ is usually between $75 \mathrm{~V}$ and $230 \mathrm{~V}$, depending on the sample, but is always less than $300 \mathrm{~V}$. The voltage is maintained at $\mathrm{V}_{\mathrm{c}}$ until the discharge is completed. When $\mathrm{V}_{\mathrm{c}}$ is low $(<100 \mathrm{~V})$ the burnt track pattern usually possesses a dense winding character, similar to that shown in Fig.2. For larger $V_{c}$, the pattern shows an outward growing radial structure near the center, followed by the winding structure at the ends. See Fig.3.

Since $\mathrm{V}_{c}$ is always less than $300 \mathrm{~V}$, in the FVM for many samples of either mineral oil or olive oil, a fixed voltage of $310 \mathrm{~V}$ is applied. The resulting pattern, shown in Fig.4, has a ramified radial structure near 


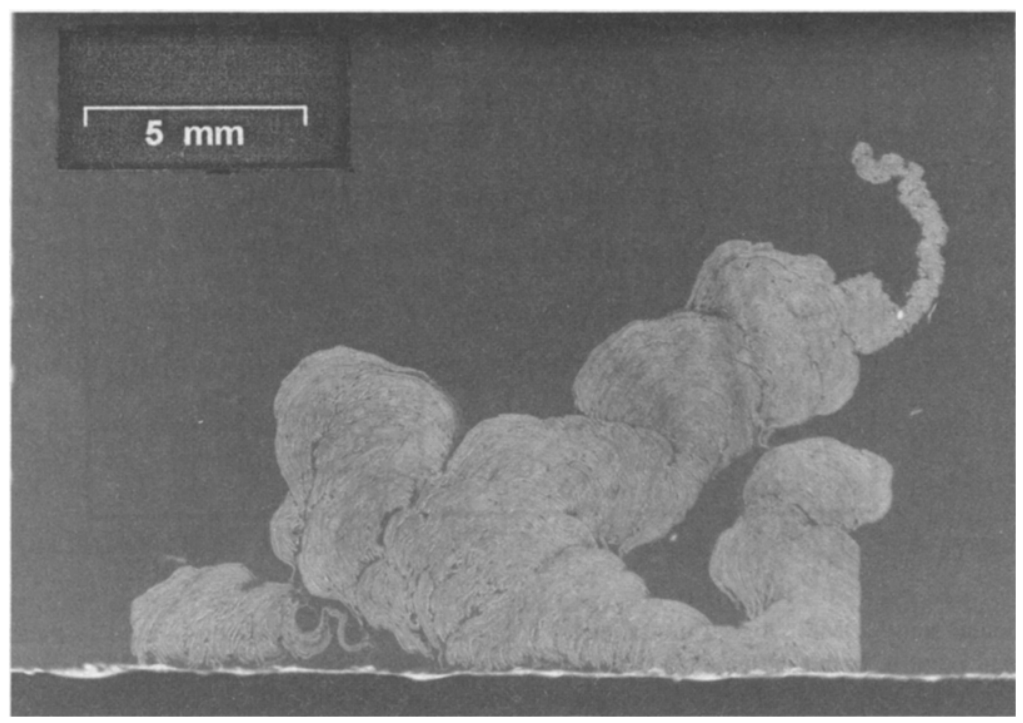

Fig. 2. Breakdown pattern with compact winding structure, obtained in an olive oil sample with the IVM and $\mathrm{V}_{c}=75 \mathrm{~V}$. The pattern shown is from the positive-voltage plate, obtained after the cell is opened and the black ashes along the tracks are cleaned away using detergent. The corresponding pattern on the negative-voltage plate is the same.

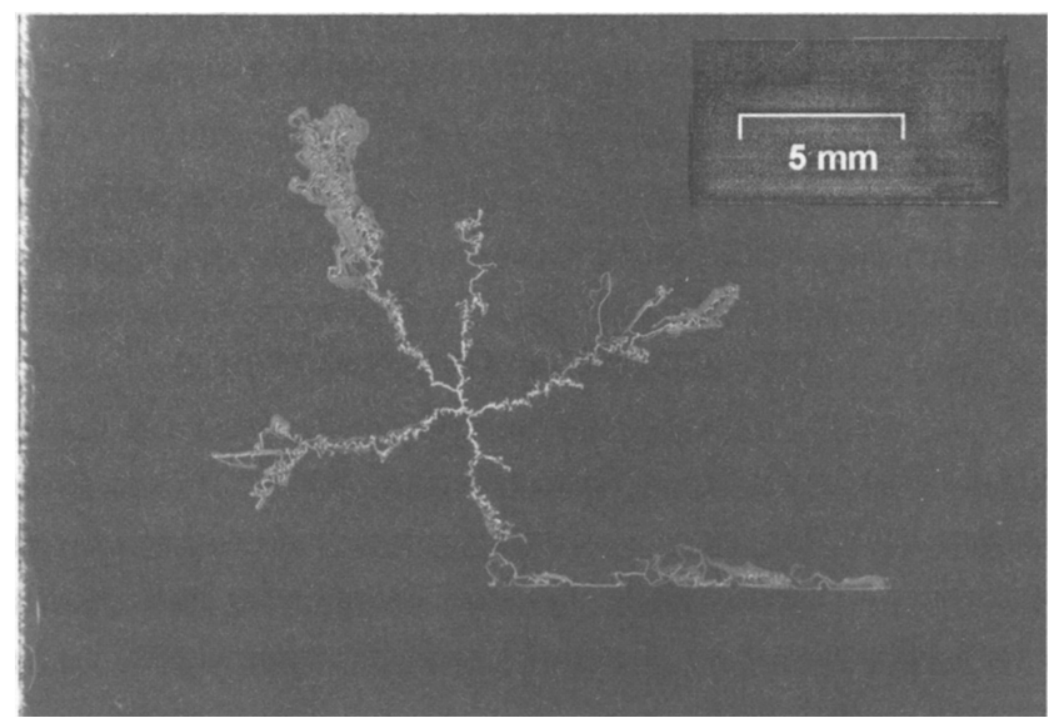

Fig. 3. Breakdown pattern with radial structure near center, obtained in a mineral oil sample with the IVM and $\mathrm{V}_{\mathrm{c}}=190 \mathrm{~V}$. Pattern shown is from the negative-potential plate. Pattern on the positive-potential plate is the same but the tracks there are thicker. 


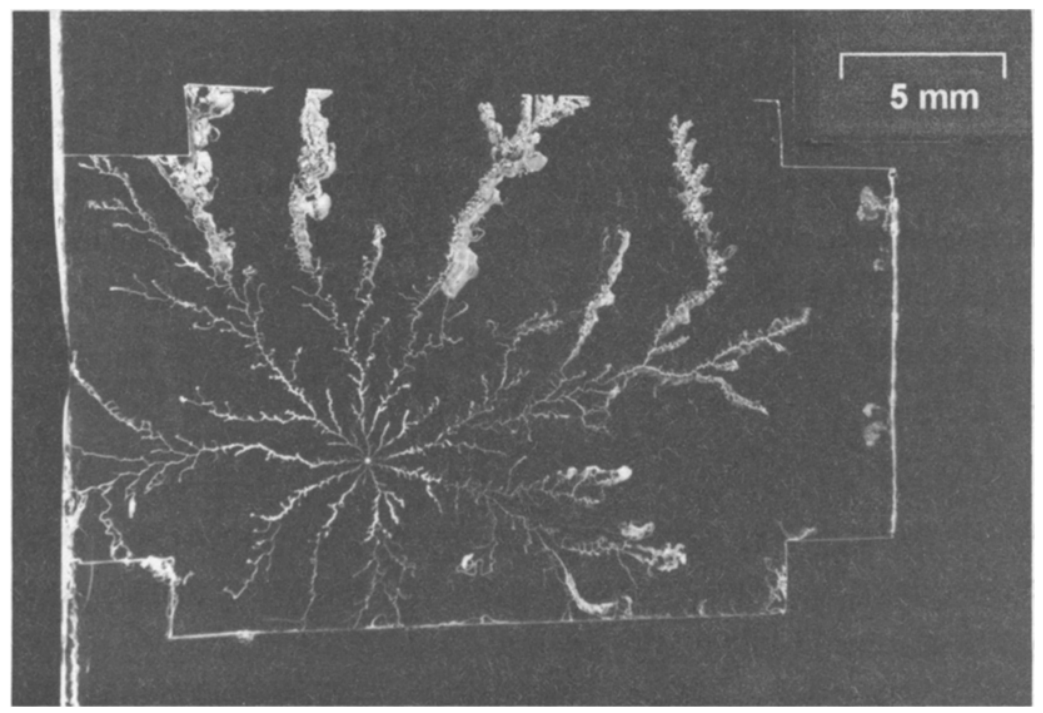

Fig. 4. Ramified radial breakdown pattern obtained in a mineral oil sample with the FVM and the fixed voltage is $310 \mathrm{~V}$. Pattern shown is from the positive-potential plate. Pattern on the negative-potential plate is identical but the tracks there are thinner.

the center and ends with a winding structure. This winding structure is similar to the high $V_{c}$ patterns obtained with the IVM. Note that in Fig.4 the thickness of the filaments alternates between thin and thick starting from the center. An enlargement of a major branch in Fig. 4 under an optical microscope is shown in Figs. 5 and 6. In Fig. 5, note the existence of a small ring (corresponding to the center of the pattern in Fig. 4) from which several branches start their growth.

In general the pattern left on the negative-potential plate, the cathode, is thinner than that on the positive-potential plate, the anode. This was also found in Ref. [10]. Comparing mineral oil and olive oil, the patterns are similar except that the filaments are thinner in the case of olive oil.

\section{VOLTAGE AND CURRENT MEASUREMENTS}

The voltage and the current are recorded by a digital storage oscilloscope. Figure 7 is the record for an IVM experiment. The breakdown voltage $\mathrm{V}_{\mathrm{c}}$ is $64 \mathrm{~V}$. The discharge time is about $0.4 \mathrm{~s}$. Due to the triggering and delay of the oscilloscope, the first data point is taken at half division after the discharge. In this run, it is $0.05 \mathrm{~s}$. This example corresponds to a low voltage breakdown, like the one in Fig.2. The current is not high enough to melt the fuse.

In the IVM, when $V_{c}$ is high $(>200 \mathrm{~V}$ ), the pattern and the time dependence of current are similar 
to that in a FVM experiment, in which the voltage is fixed at $310 \mathrm{~V}$ from the beginning. We thus turn to the FVM results.

When a fuse of $0.5 \mathrm{~A}$ is used, discharge stops when the fuse is melted. Figure 8 shows a typical example from a mineral oil cell using the FVM. The voltage becomes zero when the fuse is melted at about $12.6 \mathrm{~ms}$; at this moment the current also drops to zero. The current at the beginning is high, which decreases gradually to a plateau-like region until the fuse is melted. In another case, when a $1 \mathrm{~A}$ fuse is used, discharge starting from a point away from the edge of the cell will reach the cell edge and then stops, with the fuse not melted. The discharge time is about $50 \mathrm{~ms}$.

We found that the winding structure occurs at either a low-voltage, low-current breakdown or at the final stage of a high-voltage breakdown, where the current is also low as can be seen from the current-time record. We therefore conclude that the winding structure corresponds to a continuous slow discharge, while the radial, wiggling structure corresponds to a fast discharge.

\section{THE PHYSICAL PROCESSES}

The breakdown field strength obtained in our experiments is between $1 \times 10^{7} \mathrm{~V} / \mathrm{m}$ and $4 \times 10^{7} \mathrm{~V} / \mathrm{m}$. This is consistent with the results from other experiments using similar liquids and parallel-plate electrodes [10,17] (see also Refs. [7,8]).

The physical mechanisms behind DB in liquids are not completely understood [7,8]. The mechanisms seem to involve, but not necessarily restricted to, the initial emission of electrons from the cathode, impact or thermal ionization in the liquid bulk, and formation of gas bubbles. For the DB of a thin layer of oil under a transverse uniform electric field (perpendicular to the electrode plates) considered here, our understanding of the physical picture--based on the present and previous [10-12] experiments--is as follows. The fact that the discharge starts at some unpredictable point away from or at the edge of the cell indicates that the initial breakdown is influenced by some uncontrollable factor such as impurities or inhomogeneities of the cell, but not electron emission from the cathode. This assertion is reinforced by our experimental observation that when a small gold dot (of thickness $0.2 \mu \mathrm{m}$ ) is deposited to one of the inner surfaces of the cell, DB still starts from somewhere else (the cell edge in one experiment) but not at the gold dot even though the electric field there is the highest.

Once the discharge starts (when the voltage reaches or is above the breakdown threshod) the electrons move towards the anode while the positive ions move towards the cathode. During this process, more electrons and ions could be generated due to impact; and the local temperature of the oil increases, which also causes the vapor pressure of the oil to increase. At the location where the discharge occurs, chemical reaction(s) between the liquid and the coating material of the inner surfaces (the "burning" process) happens, leaving some black ashes behind. The fact that the burnt tracks left on the cathode and anode inner surfaces, 


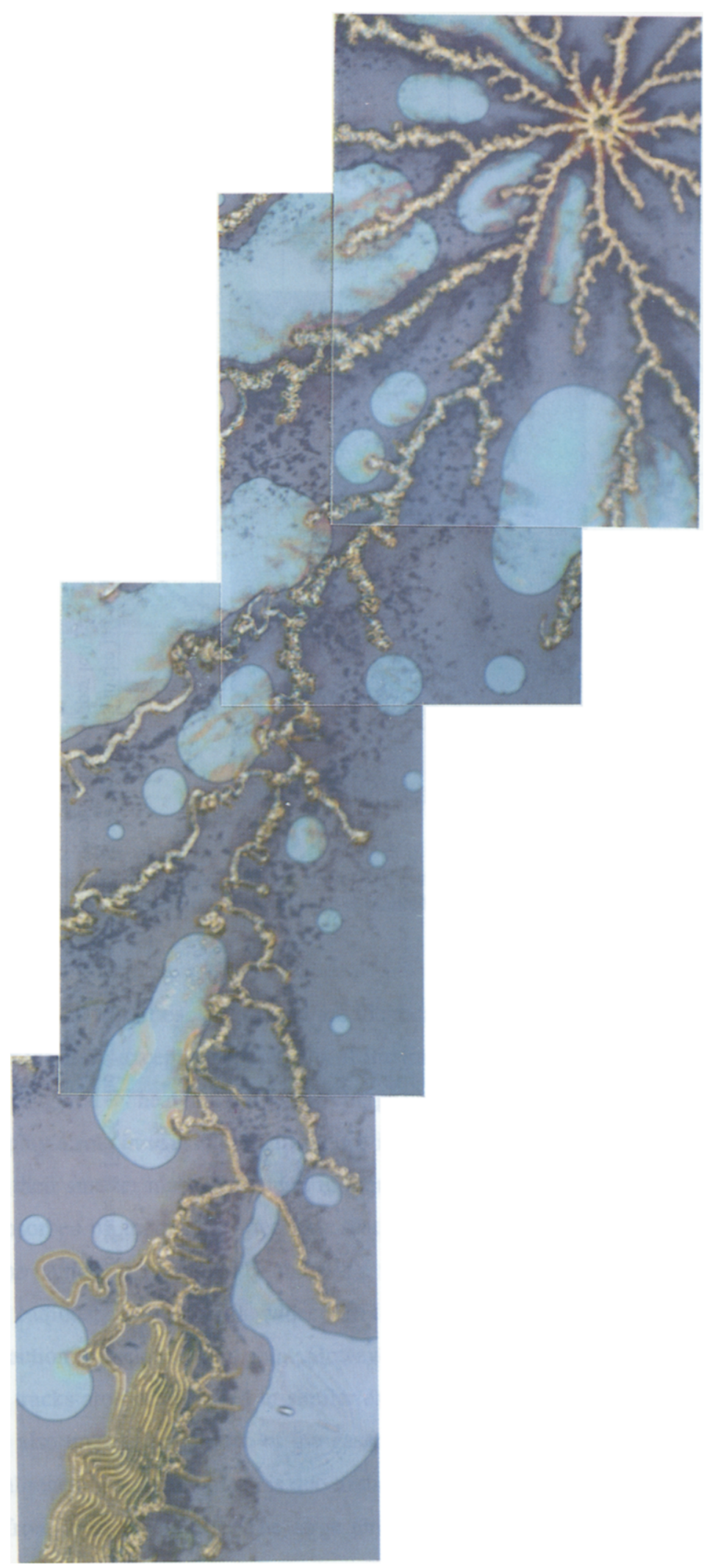




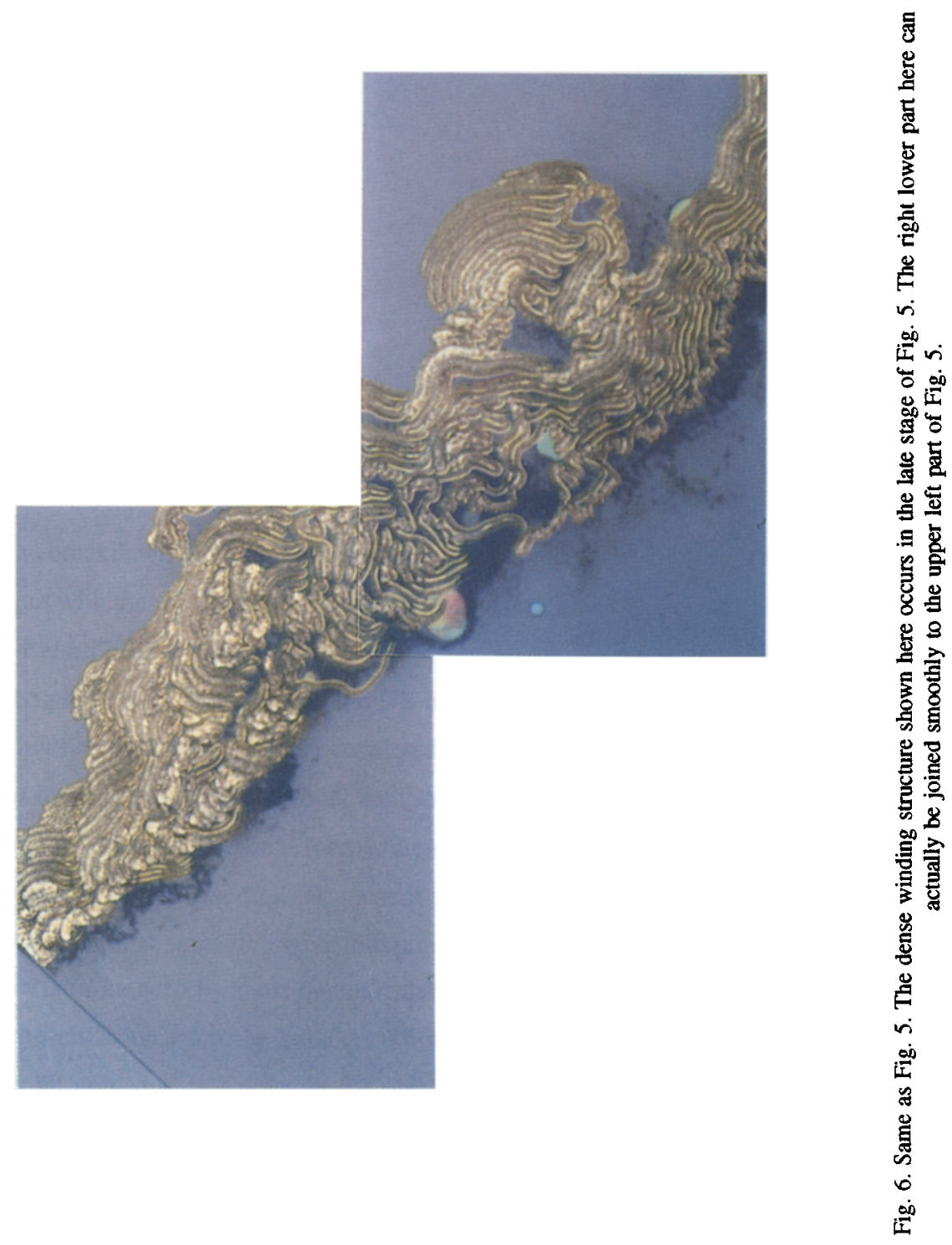




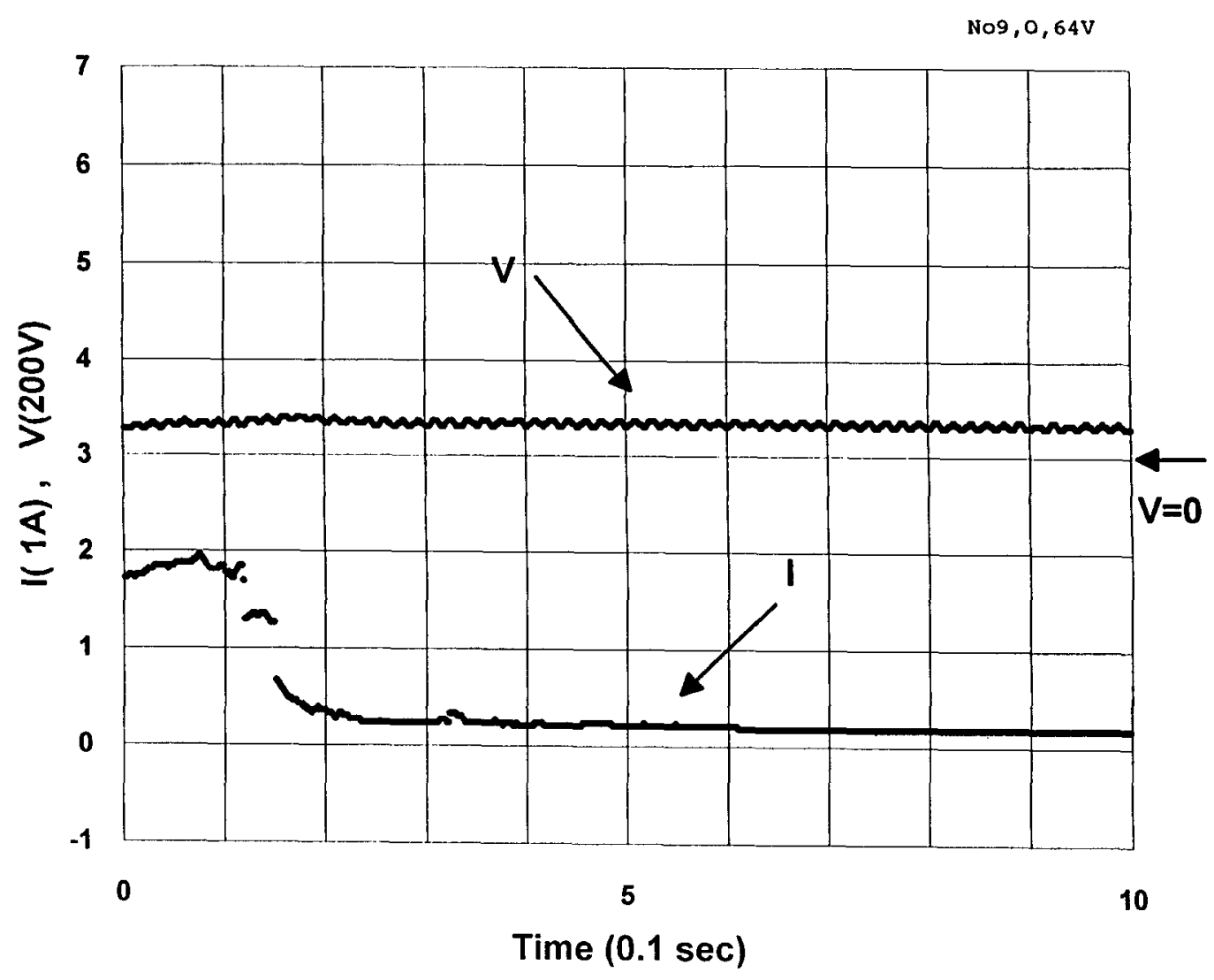

Fig. 7. Time dependences of voltage $V$ (upper curve) and current I (lower curve) obtained with the IVM and low breakdown voltage. The zero line for voltage is marked with an arrow at the right edge of this figure.

respectively, are on top of each other and are identical--in color and conformation (as examined by opening the cell after the DB), except for the widths of the tracks (see Ref. [10])--suggests that the chemical reaction is probably triggered by heat and not by the charge carriers (i.e., the electrons and ions). However, the charge carriers do play a role in causing the difference in the track width. Since electrons are more mobile than the ions due to their smaller mass, the lateral motion of the electrons (just before they reach the anode and, for those not absorbed by the anode, after their scattering from the anode) has a larger range in space than that of ions at the cathode. Consequently, there is a wider spread of the high temperature region near the anode than at the cathode, resulting in a wider burnt track on the anode as observed. The exact nature of the chemical reaction is unclear at this point. However, it seems that the organic nature of the liquid is important, since burnt tracks are not observed in similar experiments when the oil is replaced by water or air [18].

It is also unclear how much of the gas produced, as evidenced by the bubbles in the cell, is due to the chemical reaction and how much is due to the vaporization of the oil. (Since the cell is open, gas bubbles can result from local oil boiling. The large circular gas bubble covering almost the whole region of DB 


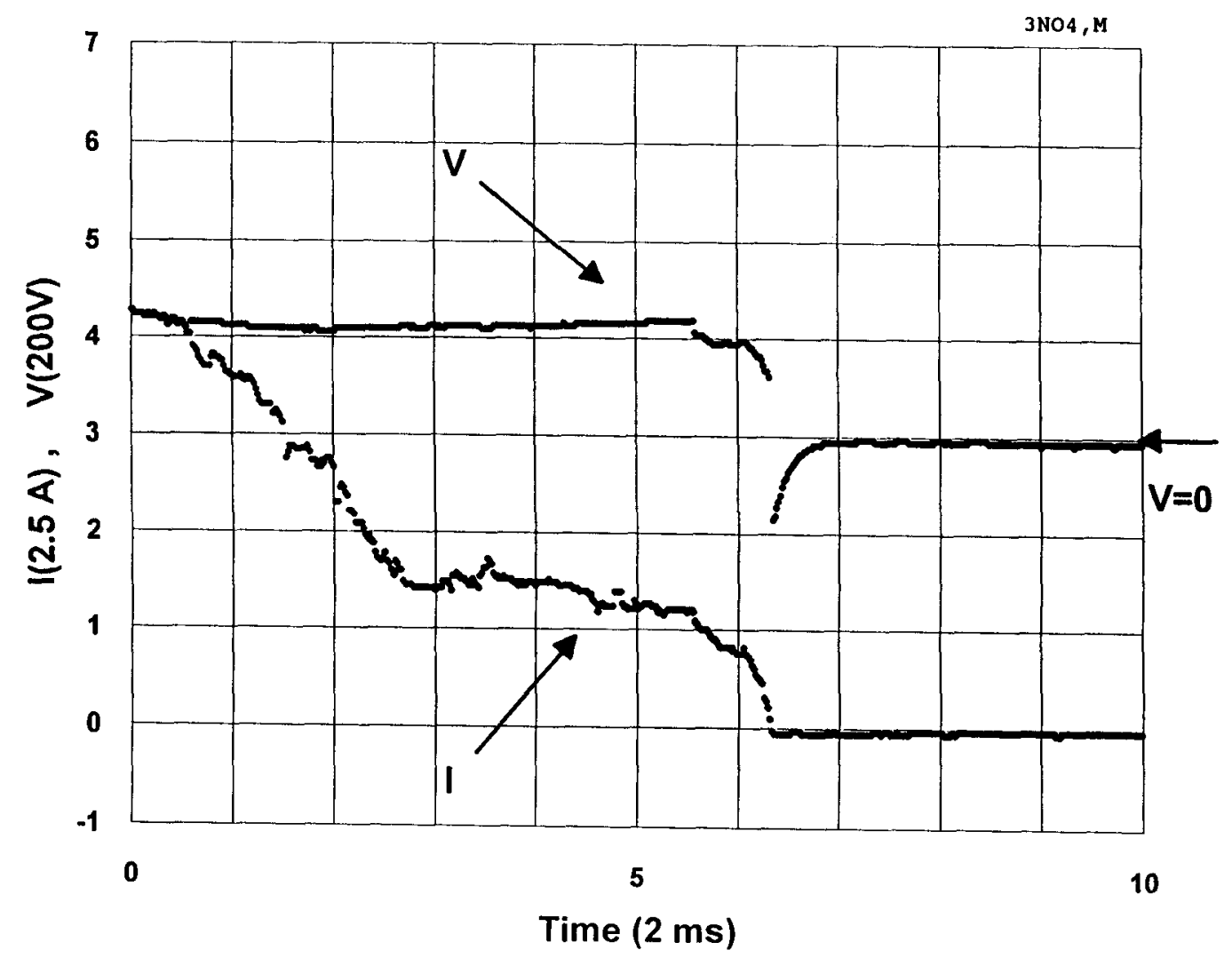

Fig. 8. Time dependences of voltage V (upper curve) and current I (lower curve) obtained with the FVM. The zero line for voltage is marked with an arrow at the right edge of this figure.

observed in a nematic MBBA liquid crystal cell [12] is very likely due to this mechanism.) In any case, the gas produced does increase the chance for discharge, due to the longer mean free paths of the charge carriers in a gas than in a liquid. The increased vapor pressure at high temperature also favors the discharge process.

Note that a filament is anisotropic in the plane of the cell, while thermal diffusion in a liquid or gas is isotropic. The formation of a DB filament thus requires a mechanism, in addition to thermal diffusion, that breaks the axisymmetry of the environment at the starting point when it is away from the cell edge. Such a mechanism, we believe, is provided by fluctuations causing the charge carriers reflected from the electrode plates to move along some selected, lateral direction(s). Once the carriers start to move in a certain lateral direction, they are immediately attracted towards the electrodes due to the transverse electric field, followed by the production of new charge carriers, chemical reactions, and reflections at the electrodes--all at this new "spot." The selected direction leading to this new spot represents the lateral thrust direction of the carrier stream, and is favored again in the next moment, giving rise to a filament with a forward-bias unlike the track of a random walk. It should be emphasized that the sequence of "spots" making up the filament are actually continously distributed, as can be seen in Fig. 5. The wiggling of the filament is due to thermal 
fluctuations, and the deformation of the gas bubbles caused by the movement of the liquid between them.

For a slow discharge, which occurs at low voltage with a low current, there is more time for heat to diffuse away from the discharge track and only the areas close to the track, surrounded by gas and vapor, are warm enough for further chemical reaction to take place. Therefore, subsequent discharge is likely to occur in the area around the existing track; a dense winding, smooth track is then developed (see Fig. 2).

On the other hand, a fast discharge occurs at the beginning of a high-voltage breakdown. Compared to the previous case, the current is larger, the heating is faster and the temperature is higher in a much larger area. In addition, the lateral thrust of the charge carriers is more intense. Consequently, (i) the next discharge can and tends to occur in the forward direction away from the existing track; (ii) accidental branching of the carrier stream, with each branch still strong enough to induce chemical reaction, may happen. A radially outward-growing track with branching is thus formed (see Figs. 3 and 4). In this fast process, the number of charge carriers reflected from the electrodes decreases in succession, since there is less time for new carriers to be generated before the reflection. Hence, as the growth of a filament is guided by the reflected charge carriers, there is a decrease of the thrust strength of the carrier stream and the fast process degenerates into a slow process as the filament grows in length. At this stage, the filament may go into the winding pattern or simply die out (see Figs. 5 and 6).

Treating the cell as two parallel-plate capacitors in parallel--one contains the burnt tracks (and possibly the surrounding gas bubbles) and the other contains the liquid parts--the surface charge density on each electrode plate is given by $\sigma=\sigma_{\mathrm{t}}+\sigma_{\mathrm{l}}$, where $\sigma_{\mathrm{l}}=\kappa \varepsilon_{0} \mathrm{~V} / \mathrm{d}$ is the liquid part and $\sigma_{\mathrm{t}}$ the track part. Here $K$ is the dielectric constant of the liquid, $V$ is the voltage across the electrodes, and $d$ is the cell thickness.

For the case of Fig. 8, $\sigma_{1}$ is estimated to be $-10^{-3} \mathrm{C} / \mathrm{m}^{2}$ which, as we will show below, is much smaller than $\sigma_{r}$. Under the assumption that the current $I$ is due to charges collected along the tracks, $I$ is given by

$$
I=N w v \sigma_{t}
$$

where the total number of active tracks $\mathrm{N}$ is given by $\mathrm{N}=\mathrm{N}_{\mathrm{g}}-\mathrm{N}_{\mathrm{d}} ; \mathrm{N}_{\mathrm{g}}\left(\mathrm{N}_{\mathrm{d}}\right)$ is the number of growing (dying) tracks; $w$ is the average width of a track; $v$ is the growth velocity of the track. For simplicity, let us assume that $w, v$, and $\sigma_{t}$ are all constant in time. Then I will increase with time when branching occurs, will decrease when some tracks die out, and will be stationary when the number of growing tracks remain constant. Thus, in Fig. 8, the generally decreasing part of $\mathrm{I}$ for $\mathrm{t}<5.5 \mathrm{~ms}$ corresponds to an overall decrease of $\mathrm{N}$, with occasional branching and dying of some tracks--in consistence with the early (before any winding occurs) portion of the pattern, as shown in Fig. 4. By the same reasoning, the plateau-like part of $I$ between -5.5 $\mathrm{ms}$ and $-12 \mathrm{~ms}$ corresponds to the outer portion in Fig. 4, the late stage dominated by track winding. (As can be seen from Fig. 6 , a winding track neither branches nor dies, resulting in $N=N_{g}=$ const. and hence $I=$ const.) With these understandings, $v$ can be estimated to be $v=5.75 \mathrm{~mm} / 5.5 \mathrm{~ms} \approx 1 \mathrm{~m} / \mathrm{s}$, where 5.75 
$\mathrm{mm}$ is approximately the radius of the inner portion of Fig. 4. From Fig. 5 , at time $t \approx 0$, one finds $\mathrm{N}_{\mathrm{g}} \approx 10$ and $N_{d}=0$, and hence $N \approx 10$. Using $w \approx d=6 \mu \mathrm{m}$ (see Sec. 6), and $I \approx 10.7 \mathrm{~A}$ at $\mathrm{t}=0$ from Fig. 8, Eq. (1) gives $\sigma_{\mathrm{t}}(\mathrm{t}=0) \approx 1.78 \times 10^{5} \mathrm{C} / \mathrm{m}^{2}$. Thus $\sigma_{\mathrm{t}} \gg \sigma_{1}$, justifying the same assumption used in Eq. (1).

The data in Fig. 7 can be understood similarly. In this case of low-voltage breakdown, the pattern consists of winding tracks (see Secs. 3 and 4). In the I curve of Fig. 7 the plateau-like region (starting from $t=0$ ) lasts a long time of $-0.12 \mathrm{~s}$, implying a very long track created during this period; which is consistent with the interpretation that a plateau in the I curve represents a winding structure.

When a leaking cell (i.e., one having electric short) is used, the DB pattern observed resembles that in Fig. 1 of Ref. [10], in which several long tracks with little branching coexist throughout the cell; the pattern is neither radial nor dense winding, unlike those in Figs. 2-4. In a leaking cell, points at which leaking occurs will have very high temperature once the voltage is applied. These points act as the starting points for the burnt tracks, and the discharge process is very fast--faster than the "fast" process giving rise to the radial structure discussed above.

An interesting DB pattern is obtained with the IVM when the cell is thicker, as shown in Fig.9(a). The pattern is essentially the same as the inner part (within a distance of $2.5 \mathrm{~mm}$ from the center) of the radial pattern in Fig. 4. The difference is that the radial pattern in Fig. 9(a) starts at a point closer to the cell edge; the pattern growth terminates before it has the chance to develop further and larger. [Even though the breakdown field strength $\left(\equiv \mathrm{V}_{c} / \mathrm{d}\right)$ in Fig. $9(\mathrm{a})(15.92 \mathrm{~V} / \mu \mathrm{m})$ is closer to that in Fig. $2(12.50 \mathrm{~V} / \mu \mathrm{m})$ than in Fig. $3(31.67 \mathrm{~V} / \mu \mathrm{m})$, however, since the cell thickness is different in the case of Fig. 9(a), the discharge process in the thick cell may still be a fast process. More experiments on cells of different thicknesses are needed to clarify this point.]

In summary, there exist three different regimes in the discharge process: (i) very fast ( $\mathrm{v}>1 \mathrm{~m} / \mathrm{s})$--the track is long with little branching; the pattern is random, covering a lot of space; (ii) fast $(\mathrm{v}-1 \mathrm{~m} / \mathrm{s})-$ the track wiggles and has more branching; the pattern is radial and ramified; and (iii) slow ( $\mathrm{v} \ll 1 \mathrm{~m} / \mathrm{s})$--the track winds and is pretty smooth; the pattern is dense winding.

\section{DISCUSSIONS}

Based on the physical processes described in Sec. 5, a burnt track is created analogously to the track of a heat-seeking missile (HSM) in bad weather [15]. As a HSM moves in space it releases heat, which diffuses with time; the motion of the missile is directed towards the region of higher temperature; the missile slows down due to air friction; and gurgles in the bad weather, causing its track to wiggle. Referring to the burnt track, the missile corresponds to the growing end of the burnt track; the heat released is due to the DB, and the chemical reaction if the latter is exothermic; the burnt track moves towards hot spots because the 


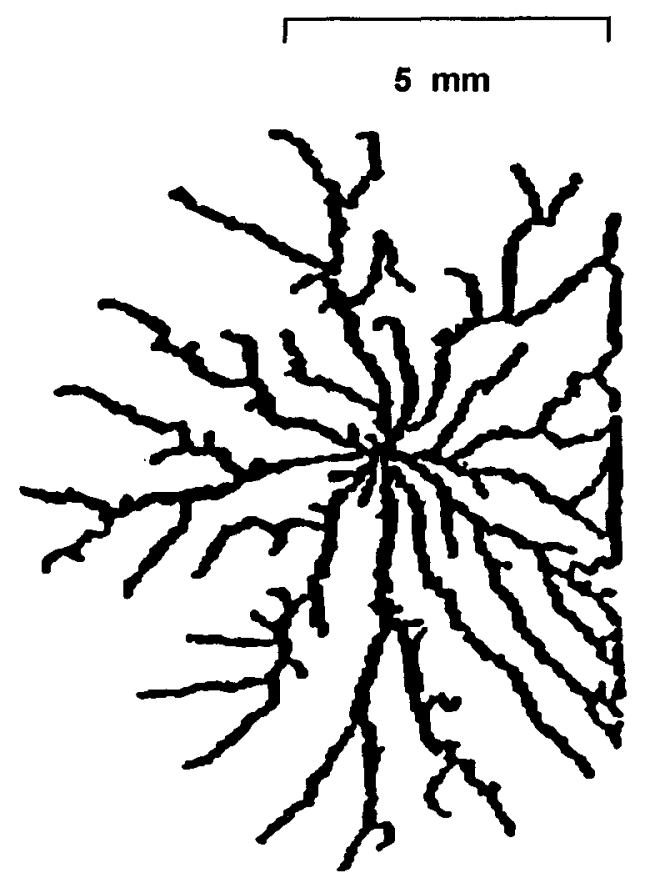

(a)

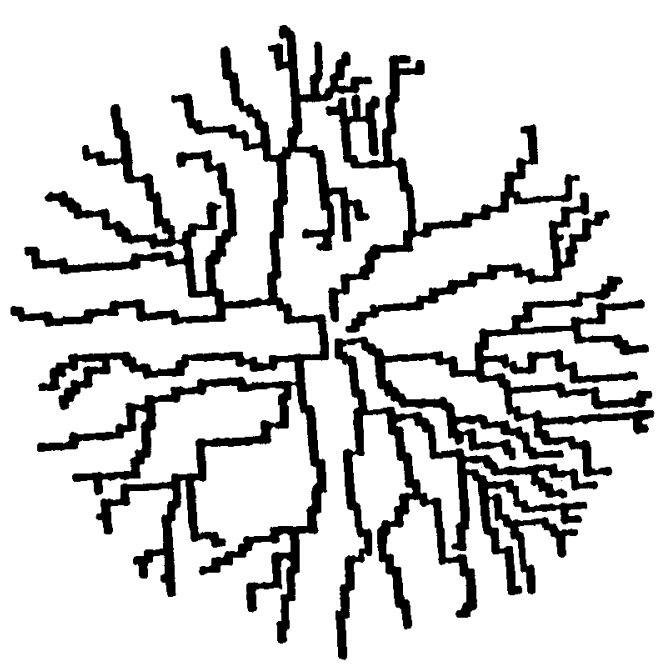

(b)

Fig. 9. (a) Breakdown pattern with radial structure, obtained in a $25 \mu \mathrm{m}$ thick olive oil sample with the IVM and $\mathrm{V}_{c}=398 \mathrm{~V}$. Pattem shown is from the positive-potential plate. Pattern on the negative-potential plate is the same but the tracks there are thinner. (b) A pattern from AWM simulation.

chance of chemical reaction occurring there is higher; the forward-bias of the track corresponds to the inertial motion of the missile; the bad weather represents thermal fluctuations; etc. However, the analogy is not exact. The major difference between a HSM and a burnt track is that self-crossing of the track is allowed in the former but not in the latter case (as can be seen in Figs. 2-4, implying that the burning process is pretty thorough; after the burning there is no or insufficient coating chemical left there for a second burning.)

The burnt track formation is thus represented by a heat-generating, heat-seeking "particle" coupled to a temperature field. The coupled equations are given by

$$
m d^{2} \mathbf{R} / d t^{2}=-\eta(T) d R / d t+\alpha(T) \int d \mathbf{r} \delta(\mathbf{r}-\mathbf{R}) \nabla T(r, t)+\mathbf{F}(t)
$$

and

$$
\partial \mathrm{T}(\mathbf{r}, \mathrm{t}) / \partial \mathrm{t}=\chi \nabla^{2} \mathrm{~T}(\mathbf{r}, \mathrm{t})+\gamma(\mathrm{T}) \delta(\mathbf{r}-\mathbf{R})
$$

where $m$ and $\mathbf{R}$ are, respectively, the mass and position of the particle; $T$ is the temperature. The damping coefficient $\eta, \alpha$, and the heat production coefficient $\gamma$ are all positive; $\eta$ and $\gamma$ may depend on the external 
electric field; $\chi$ is the thermal diffusivity; $\mathbf{F}$ is a random force. Note that self-avoidance and branching of the track are absent in Eqs. (2) and (3). [Equations (2) and (3) are similar but not identical to those used to describe the clustering of active walkers [19].] Depending on the coefficients, the track of the particle may terminate or grow forever, be localized or extended.

Equations (2) and (3), or the related Fokker-Planck equations, are difficult to solve. In order to compare with real DB patterns, computer modeling (with self-avoidance and branching easily included) has been used [11-13]. The computer model constructed is the AWM, in which the particle in Eq. (2) is an active walker capable of changing the landscape--a potential related to $-T$ and the deficiency of coating materials. (See Refs. [14,15] for details.) One of the patterns [11] generated by our AWM is shown in Fig. 9(b), which agrees very well with the DB pattern shown in Fig. 9(a). The fact that the pattern in Fig. 9(b) also closely resembles the retinal neurons [12], a biological system very different from the physical/chemical example of $\mathrm{DB}$ considered here, indicates that the AWM is a pretty universal model in pattern formation.

In Sec. 5, the estimated value of $\sigma_{t} \sim 10^{5} \mathrm{C} / \mathrm{m}^{2}$ is based on the assumption of $w \approx d$. If the width $w$ in Eq. (1) is taken to include not just the burnt track but also some bubble area surrounding the track, then w may be in the millimeter range (see Figs. 4 and 5) and $\sigma_{t}$ would be three orders of magnitude smaller, which is still much greater than $\sigma_{1}$. Further experiments with the growth of the tracks photographed in real time will be very useful in telling the locations and sizes of the gas bubbles. When correlated with the time records of $I$ and $V$, a sequence of pictures showing the track growth will enable us to determine $w, v$, and hence $\sigma_{\mathrm{t}}$ as functions of $\mathrm{t}$, with the use of Eq. (1). Many aspects of the physical mechanisms can be ascertained if a single track can be created far from the cell edge.

In the experiments of Atten and Sekar [16], a tungsten needle is placed very close $(<100 \mu \mathrm{m})$ to a glass disk coated with indium oxide on the side away from the needle. Both electrodes are immersed in mineral oil. The radial DB pattern, videoed from the bottom of the transparent glass disk, is not strictly twodimensional; it is in fact a projection of the three-dimensional pattern on the glass plate. In this point-plane setup, the electric field is almost parallel to the glass plane and the DB tracks run in the direction of the electric field. In contrast, in our experiments, the tracks are perpendicular to the electric field; the mechanisms of track formation are obviously different. If the coated glass disk in Ref. [16] is flipped $180^{\circ}$ so that the needle now faces the coating, the resulting DB pattern would be similar to that observed before but burnt tracks, due to chemical reactions similar to those in our experiments, could be left on the coated side of the glass disk--a permanent record of the DB pattern results.

Recently, experiments similar to ours have been carried out by Ince et al [20]. When silicone oil, instead of mineral oil, is used similar patterns are obtained. However, when the indium tin oxide coating on the glass plates is replaced by aluminum coating, slightly different patterns are observed. 


\section{REFERENCES}

Nonlinear Structures in Physical Systems, edited by L. Lam and H.C. Morris. Springer, New York (1990).

Pattern Formation in Complex Dissipative Systems, edited by S. Kai. World Scientific, River Edge (1992).

Spatiotemporal Patterns in Nonequilibrium Complex Systems, edited by P.E. Cladis and P. Palffy-Muhoray. AddisionWesley, Redwood City (1994).

Introduction to Nonlinear Physics, edited by L. Lam. Springer, New York (1995).

See, e.g., the series, Progress in Dielectrics.

I. Niemeyer, L. Pietronero, and H.J. Wiesmann, Fractal Dimension of Dielectric Breakdown, Phys. Rev. Lett. 52, 10331036 (1984).

I. Adamczewski, Ionization, Conductivity and Breakdown in Dielectric Liquids. Taylor \& Francis, London (1969).

T.J. Gallagher, Simple Dielectric Liquids: Mobility, Conduction, and Breakdown. Oxford University Press, Oxford (1975). J.C. Devins, S.J. Rzad, and R.J. Schwabe, Breakdown and Prebreakdown Phenomena in Liquids, J. Appl. Phys. 52, 45314545 (1981).

L. Lam, R.D. Freimuth, and H.S. Lakkaraju, Fractal Patterns in Burned Hele-Shaw Cells of Liquid Crystals and Oils, Mol. Cryst. Liq. Cryst. 199, 249-255 (1991).

L. Lam, R.D. Freimuth, M.K. Pon, D.R. Kayser, J.T. Fredrick, and R.D. Pochy, Filamentary Patterns and Rough Surfaces, in Pattern Formation in Complex Dissipative Systems, edited by S. Kai, pp. 34-46. World Scientific, River Edge (1992). D.R. Kayser, L.K. Aberle, R.D. Pochy, and L. Lam, Active Walker Models: Tracks and Landscapes, Physica A 191, 17-24 (1992).

14. L. Lam and R.D. Pochy, Active Walker Models: Growth and Form in Nonequilibrium Systems, Comput. Phys. 7, 534-541 (1993).

15. L. Lam, Active Walker Models for Complex Systems, Chaos Solitons \& Fractals, this issue.

16. P. Atten and A. Saker, Streamer Propagation over a Liquid/Solid Interface, IEEE Trans. Elect. Ins. 28, 230-242 (1993).

17. G.S. Kuchinskii, Theory of Breakdown of Thin Liquid Dielectric Layers, Sov. Phys.-Tech. Phys. 11, $964-969$ (1967).

18. L. Lam and R.D. Freimuth, unpublished.

19. F. Schweitzer and L. Schimansky-Geier, Clustering of Active Walkers in a Two-Component System, Physica A (in press).

20. A. Ince, R. Runge, and K. Wantanabe, The Observation and Computer Simulation of Fractal Pattern Formation in Dielectric Breakdowns of Liquids. Thesis, Department of Physics, University of California at Riverside (1994). 